\title{
Cuvierian Functionalism
}

\begin{abstract}
Aaron Novick*
The rise of evolutionary-developmental biology (evo-devo) is often claimed to mark the return of typological and/or structuralist thinking to evolutionary biology. Proponents of such claims point to evo-devo's emphasis on the deep conservation of animal body plans. Deep conservation requires long-term evolutionary stasis, which is difficult to explain in a Darwinian functionalist framework. Evo-devo biologists must therefore rely on other argumentative strategies to explain deep conservation. I argue, however, that their explanations are not typological or structuralist. Rather, they are Cuvierian functionalist: they recapitulate, in modified form, an argument developed by Georges Cuvier in the nineteenth century. Cuvierian functionalism is distinct from but complementary to Darwinian functionalism. Moreover, Cuvierian functionalism is immune to the traditional objections to typological thinking.
\end{abstract}

\section{Keywords}

cuvierian functionalism $\bullet$ evo-devo $\bullet$ typology $\bullet$ population thinking $\bullet$ Cuvier, Georges $\bullet$ gene regulatory networks $\bullet$ deep conservation

Cuvier believed that the radically different organizations of the four embranchements could not change from one to another without a fatal loss of integrated function during the transition. This argument is still a forceful one.

- Rudolf Raff (1996, 6)

\section{Anti-Gradualism, Then and Now}

This paper makes the case that evolutionary-developmental biology (evo-devo), in explaining the deep conservation of animal body plans, relies on a Cuvierian functionalist explanatory strategy. Philosophical analysis commonly treats evo-devo as a "typological" research program (Alberch 1989; Amundson 2005; Brigandt 2007; Lewens 2009), in contrast to the population thinking that undergirds population-genetic approaches to evolutionary theorizing (Mayr

* Department of Biochemistry and Molecular Biology, Department of Philosophy, Dalhousie University,

Tupper 8-A2, 5850 College St, Halifax, NS B3H 4H7, Canada, aaron.novick@dal.ca,

(D) https://orcid.org/0000-0001-5081-7527

Received 30 November 2018; Revised 10 January 2019; Accepted 11 January 2019 doi:10.3998/ptpbio.16039257.0011.005

๑ OPEN ACCESS - PTPBIO.ORG 
1959). The central aim of this paper is to show that many of the features that have led evo-devo to be treated as typological are in fact the product of its Cuvierian functionalism. To achieve this overarching goal, this paper has three subsidiary aims: (1) to distinguish two ways in which Mayr's notoriously unprincipled notion of typological thinking can be made precise, (2) to show that one of these ways-Cuvierian functionalism - plays an important role in evo-devo's developmental genetic explanations of the deep conservation of animal body plans, (3) to show that Cuvierian functionalism is compatible with and complementary to the Darwinian functionalism and population thinking of mainstream evolutionary theory. The remainder of this introduction is concerned with making the relations between these three aims more salient.

As Ernst Mayr tells the story, one of Darwin's major achievements was to replace typological thinking with population thinking (Mayr 1959; see also Hull 1965a, 1965b). Typological thinking, rooted in Platonic philosophy, had dominated pre-Darwinian biology, which treated species as fixed types, limited in their ability to vary. Darwin's Origin stood this way of thinking on its head, making variation fundamental and unlimited, relegating "types" to the status of statistical epiphenomena. All major disputes in evolutionary biology, according to Mayr, could be analyzed as pitting typological thinking against population thinking. The core of these disputes lay in the incompatibility of typological thinking and acceptance of gradualistic evolutionary theories. A typologist, for Mayr, was necessarily an anti-gradualist.

As evo-devo has matured over the past several decades, it has frequently found itself at odds with mainstream evolutionary theorizing. In attempting to understand these tensions, scholars have latched onto Mayr's framework. Both evo-devo biologists (Alberch 1989; Wagner 2014) and sympathetic philosophers (Amundson 1998; Brigandt 2007; Lewens 2009; Love 2009) have argued that typological thinking is essential to, and therefore vindicated by, evo-devo's successes. As every modus ponens has its modus tollens, so some critics have suggested that evodevo is vitiated by its typology (Jenner 2006). Ron Amundson (2005, chap. 11) has gone so far as to suggest that evo-devo's reliance on typological thinking is one of the key factors that renders it incommensurable with mainstream evolutionary theorizing, with the latter's commitment to exclusive population thinking.

A major impetus for understanding evo-devo as typological stems from its emphasis on deep conservation. This emphasis manifests at several levels, as evo-devo biologists study (i) animals' deeply conserved genetic toolkit (Carroll 2008); (ii) gene regulatory network "kernels" that have persisted unchanged for over 500 million years (Davidson and Erwin 2006; Peter and Davidson 2015, chap. 7); (iii) the conservation of complex phenotypic arrangements (Wagner and Schwenk 2000; Schwenk and Wagner 2001); and (iv) the deep conservation of animal body plans (Raff 1996; Levin et al. 2016). Raff (1996, chap. 1) has suggested that this is a major difference between evo-devo and mainstream evolutionary theorizing: where the latter emphasizes the diversity of the organic world (cf. Dobzhansky 1982, chap. 1), the former emphasizes universality. The focus on what is constant as opposed to what is changing makes an analysis of evo-devo as typological — as a science of fixed types - alluring.

The difficulty with analyzing evo-devo as typological is that Mayr never offered a coherent account of typological thinking. As Joeri Witteveen $(2015,2016)$ has shown, Mayr's initial introduction of the concept, justified as a "temporary oversimplification", was unprincipled from the start, merging distinct concepts in a haphazard way, and only got worse with time. I am convinced by Witteveen's work that "typology" and "typological thinking" are terms beyond repair, and that the analysis of evo-devo as typological is more apt to create confusion than illumination.

I do not, however, think that the Mayrian project was entirely hopeless. In introducing the notion of typological thinking, one of Mayr's aims was to isolate a common pattern of thought 
that gives rise to opposition to gradualistic evolutionary theories. He believed he could identify a commitment common to all anti-gradualists, a commitment shared despite otherwise distinct theoretical views. Though he failed in his attempt to carry out this project, the question itself is a good one: Are there common paths to anti-gradualism, repeated throughout the history of biology? This question is especially pertinent for understanding evo-devo. The problem of deep conservation is the problem of evolutionary stasis: Deeply conserved features of organisms are just those features that have, over long periods of time and in multiple distinct lineages, failed to evolve. By understanding the various routes to anti-gradualism adopted by past biologists, we may thereby come to better understand the conceptual foundations of evo-devo.

In what follows, I argue that there are at least two distinct paths to anti-gradualism, one of which plays a crucial role in evo-devo's explanation of deep conservation. I begin by presenting Mayr's notion of typological thinking and demonstrating its close connection with antigradualism (\$2), after which I show that anti-gradualism plays a key role in evo-devo's developmental genetic explanation of deep conservation (\$3). I then distinguish two historical paths to anti-gradualism, natural state thinking (Sober 1980) and Cuvierian functionalism (\$4). Next, I show that Cuvierian functionalism plays a central role in evo-devo's explanations of deep conservation, while natural state thinking plays no such role $(\$ 5)$. Having established this, I discuss the relationship between Cuvierian functionalism and Darwinian functionalism, showing that they are distinct but complementary (\$6). I further argue that Cuvierian functionalism is compatible with a defensible interpretation of population thinking, and that versions of population thinking that deem Cuvierian functionalist reasoning illegitimate are indefensible ( $\$ 7$ ). I conclude by reflecting on how best to understand evo-devo's emphasis on universality and conservation in connection with mainstream evolutionary theorizing's emphasis on diversity (§8).

\section{Typological Thinking and Anti-Gradualism}

Mayr believed that the division between typological thinking and population thinking rested primarily on metaphysical assumptions about the nature of variation. For the typologist, there exist "a limited number of fixed, unchangeable 'ideas' underlying the observed variability" (Mayr $1959,2)$. These ideas or types are fundamental, while variation is ephemeral, and constrained within limits set by the type. For the population thinker, by contrast, unique individuals are the ontological bedrock, and any collective description of a group of individuals (e.g., a species) is only a statistical summary of the properties of those individuals. This statistical "type" is ephemeral (change the individuals, and the "type" will change with them), while individuals and their variations are fundamental.

Typological thinking, Mayr claimed, is incompatible with accepting gradual evolution:

Since there is no gradation between types, gradual evolution is basically a logical impossibility for the typologist. Evolution, if it occurs at all, has to proceed in steps or jumps. (Mayr 1959, 2)

Accepting typological metaphysics precludes accepting the possibility of gradual change between types. Because types constrain variation, only two possibilities are open to the typological thinker: (i) the complete absence of evolution beyond the boundaries set by the underlying types (i.e., species fixism), or (ii) evolution by single-step changes from one type to another (saltationism). Gradual evolution, understood by the typologist as the change from one type to another by the gradual accumulation of small, within-type variants, cannot proceed beyond the limits of type-that is, it cannot give rise to new types. For the population thinker, by contrast, no such obstacles impede the acceptance of Darwinian evolutionary theory. Because variation is 
fundamental and unconstrained by any underlying type, natural selection can accumulate small variations indefinitely.

For Mayr, typological thinking was not merely one path to anti-gradualism among many. It was the path, repeated throughout the history of evolutionary theorizing:

Indeed, all saltationists have been typologists, and most typologists have been saltationist of one sort or another. Genuine variation and gradual change are, of course, incompatible with the typological viewpoint. (Mayr 1960, 364)

Opposition to gradualism has indeed played an important role in the history of evolutionary thought. This role has been most notorious in its global forms, including both species fixists who opposed the possibility of evolution altogether (e.g., Cuvier 1831, 1836; Lyell 1832) and saltationists who reject the possibility of the gradual evolution of new species and higher taxa (e.g., Goldschmidt 1982; Schindewolf 1993). We shall also see, over the course of this paper, that localized forms of anti-gradualism have been and still are important for explaining the deep conservation of some (but not all-hence locality) features of organisms.

However, Mayr's attempt to explain this history of anti-gradualism in terms of a single, sweeping mode of thought- "typological thinking"-was a failure, for two reasons. First, typological thinking, as Mayr characterized it, fails to capture the commitments of nearly all important anti-gradualists - in the end, perhaps the only major figure who answers to Mayr's characterization of typology is Louis Agassiz (Winsor 2006). Second, as Joeri Witteveen (2015, 2016) has shown, Mayr formed his conception of typological thinking by conflating two different distinctions (owing to George Gaylord Simpson and Theodosius Dobzhansky, respectively), a conflation that preserved the usefulness of neither of his sources. Given these problems, Mayr's notion of typological thinking should be abandoned as an analytic category used to make sense of evo-devo (or any other research program).

However, even if Mayr failed in his attempt to explain all opposition to gradualism in one fell swoop, his basic project of searching for common themes underwriting anti-gradualism in its myriad incarnations is worthwhile. Valuable work has been done in this area by Elliott Sober (1980), who showed that at least one important source of anti-gradualism, which I call natural state thinking, can be made philosophically precise. We will see below that natural state thinking does not account for the role that localized anti-gradualism plays in evo-devo, but before the underpinnings of contemporary anti-gradualism can be disentangled, I must show that anti-gradualism plays an important role in contemporary evo-devo. To that task, I now turn.

\section{Explaining Deep Conservation}

Evo-devo has two features that make it tempting to analyze it as a typological research program, as numerous philosophers have done (Amundson 1998; Brigandt 2007; Lewens 2009; Love 2009). The first is evo-devo's emphasis on the deep conservation: of animal body plans (Raff 1996; Davidson and Erwin 2006), of developmental stages (Levin et al. 2016), and of a core "genetic toolkit" deployed in animal development (Carroll 2008). The second is evo-devo's invocation of these conserved features in causal explanations of evolutionary change (e.g., A1berch and Gale 1985).

Regarding the first feature, deep conservation, evo-devo takes as one of its central explananda the fact that animal body plans are both limited in number and deeply conserved (Hall 1999, 98; Amundson 2005, 235). There are about thirty basic plans of construction to which all animals can be referred (Raff 1996), and most of these body plans have been conserved since the

○ OPEN ACCESS - PTPBIO.ORG 
Cambrian radiation, over 500 million years ago (Valentine 2004; Erwin and Valentine 2013). To put the point a bit differently: if one imagines the space of all possible animal forms, or morphospace, then the occupation of morphospace by actual animals is clumpy, and these clumps have remained stable for hundreds of millions of years.

Evolutionarily conserved features are, by definition, features that have not changed over long periods of evolutionary time. To explain deep conservation is thus to explain why certain features of organisms have, in numerous different lineages across long periods of time, failed to change. This explanatory task is therefore, in a certain sense, inherently anti-gradualist. Failure to change is ipso facto failure to change gradually, and thus evo-devo must explain why these features of animals have failed to evolve gradually, even as other features of animals have changed. This localized anti-gradualist element that necessarily accompanies an emphasis on deep conservation provides one reason why it can be attractive to analyze evo-devo as a typological research program.

The second apparently "typological" feature of evo-devo is closely related to the first. Because these features of animals are deeply conserved, and so are present in numerous different lineages, it is possible to invoke them as causal influences on evolution in otherwise independent lineages. This will be discussed further below (\$7); here, I simply give a quick example. In a classic work of early evo-devo, Pere Alberch and Emily Gale (1985) attempted to explain patterns of digit loss in frogs. Several frog lineages have independently evolved digit loss phenotypes. Interestingly, despite these losses being independent evolutionary events, each lineage has lost digits (or parts of digits) in the same order. Thus, for instance, frog lineages that experience loss from a single digit always experience loss from the first toe, while lineages that experience loss from two digits always experience loss from the first and fifth toes (Alberch and Gale 1985, 10-11). Alberch and Gale explain this in terms of conserved features of frog limb development, which they argue constrain the types of digit loss phenotypes that can be generated (and thus are available to selection). Alberch and Gale treat their model of the generalized frog foot as more than a mere statistical summary: they use it to develop causal explanations of evolutionary change. This treatment of developmental types as causal factors in evolutionary processes also makes it attractive to analyze evo-devo as a typological research program.

The task, then - given the problems with "typological thinking" as an analytic category-is to determine the real conceptual foundation of these aspects of evo-devo. That is the aim of the remainder of this paper. To get started on this task, let us consider an example of how deep conservation is explained in evo-devo. Eric Davidson (a developmental geneticist) and Douglas Erwin (a paleontologist) have offered an explanation of the deep conservation of animal body plans in terms of the genetic underpinnings of development (Davidson and Erwin 2006; Erwin 2015; Peter and Davidson 2015, chap. 7). Anti-gradualism plays a crucial role in this explanation. In subsequent sections ( $\S 4-6)$, I will draw out the underlying pattern of thought involved in this explanation, but for now I simply wish to present it with as little philosophical baggage as possible.

According to gene regulatory network (GRN) theory, the orderly progress of animal development (from a single cell to a fully differentiated organism) depends on the action of hierarchically structured GRNs, which consist of genes whose protein products regulate the expression of other genes (including genes whose protein products regulate the expression of other genesthus the hierarchical structure). These networks precisely control the spatiotemporal expression of genes in the developing organism, allowing the embryo to differentiate. This process involves different types of developmental tasks, and one of the important results of GRN theory is that similar tasks tend to be accomplished by the activity of similar structured GRN subcircuits (Peter and Davidson 2015, chap. 6). For instance, in order to develop a body part, an embryo must 
(a) specify the domain in which the part will develop, (b) determine the morphological structure of the part, and (c) actually build the part. Different GRN subcircuits underlie each task (Peter and Davidson 2011).

Davidson and Erwin's explanation of deep conservation concerns the first of these tasks: domain-specification. They argue that deep conservation of body plan features depends on the deep conservation of GRN subcircuits, called "kernels", essential for domain-specification. If this is correct, then the task of explaining body plan conservation becomes the task of explaining kernel conservation. Davidson and Erwin $(2006,796)$ point to four key features of kernels that explain their deep conservation:

1. Kernels "execute the developmental patterning functions required to specify the spatial domain of an embryo in which a given body part will form." Accordingly, they are high in the GRN hierarchy, i.e., the network downstream of a kernel is much larger than the network upstream.

2. They are dedicated to serving that function alone; they are not reused elsewhere in development.

3. They are recursively wired (i.e., they involve a great deal of positive feedback), with the result that "the products of multiple regulatory genes of the kernel are required for function of each of the participating cis-regulatory modules of the kernel."

4. Because of this recursive wiring, "interference with the expression of any one kernel gene will destroy kernel function altogether," resulting in the complete failure to develop the body part in question.

Together, these four properties of kernels explain why kernels are deeply conserved. Davidson and Erwin (2006) offer two examples of kernels: one for endomesoderm specification in echinoderms, and one for heart-field specification conserved between Drosophila and vertebrates (see Peter and Davidson 2015, chap. 7 for further examples). Both of these kernels originated when Bilateria first diversified ( $>500$ million years ago) and have been conserved since. What explains this? Davidson and Erwin (2006, 799) write:

We think that change in [kernels] is prohibited on pain of developmental catastrophe, both because of their internal recursive wiring and because of their roles high in the developmental hierarchy.

Because kernels are recursively wired, each gene in the kernel is essential to its function. Eliminating any one gene disrupts the functioning of the entire kernel. Because kernels are high in the developmental GRN hierarchy, such disruption has substantial downstream consequences, i.e., kernels are highly generatively entrenched (Schank and Wimsatt 1986; Wimsatt 2015). Disrupting the heart progenitor field specification kernel, for instance, prevents the development of a heart. Thus, any change to the linkage in a kernel is likely to have catastrophic phenotypic effects. This generates strong selection against mutations that cause such changes-heartless

\footnotetext{
${ }^{1}$ In fact, there are some differences between the heart-field specification kernel in Drosophila and mouse (Davidson and Erwin 2006). These changes are the exceptions that prove the rule. Each of the changes is of the same type: what is a direct linkage between two genes in one species is an indirect linkage in the other. For instance, in mouse, Gata4 activates Nkx2.5 directly, whereas in Drosophila Pnr (Gata4 homolog) activates Mid, which activates Tin (Nkx2.5 homolog). The regulatory result is the same: gene $A$ activates gene $B$. The only difference concerns the exact route by which this result is achieved.
} 
animals don't leave many offspring. That leads to the deep conservation of kernels, and thus to the deep conservation of those body plan elements that depend on them.

Three features of this explanation deserve mention, as they will play a large role in what follows. First, it is not an explanation of the origin of GRN kernels. It is an explanation of the conservation of GRN kernels that have already originated. Davidson and Erwin have little to say about the initial formation of kernels, and wisely so, since the processes by which kernels formed lie in the deep past and are epistemically inaccessible with current research methods. They simply assume that kernels are produced by gradual, Darwinian evolution of a mundane sort (Davidson and Erwin 2006, 799). As such, their explanation is explicitly not saltationist (Erwin and Davidson 2006; contra Coyne 2006).

Second, a crucial premise of their argument is anti-gradualist, albeit in a local and not a global sense. They claim that there is no possibility of gradual phenotypic evolution through the modification of GRN kernels, because no changes to kernel topology produce small phenotypic effects. GRN kernels can thus either evolve by saltation or not at all. Since the large phenotypic changes that result when kernel topology is modified are generally lethal, the result is that GRN kernels, once formed, do not subsequently change. This is not a rejection of gradual evolution altogether-as we have just seen, they allow for the gradual origin of kernels, as well as the gradual evolution of other portions of the GRN hierarchy. It is merely an argument that a particular type of GRN subcircuit cannot evolve gradually once formed. It is thus a form of local, rather than global, anti-gradualism.

Third, the anti-gradualism involved in their explanation of deep conservation is not absolute. GRN kernels represent one extreme in a graded hierarchy of variability and conservation. Davidson and Erwin (2006, fig. 3) suggest a general picture according to which different levels of the GRN hierarchy tend to correspond (roughly) to features conserved at different levels of the Linnean hierarchy: kernels underlie characters conserved at or above the phylum level, while differences at the periphery of the network tend to underlie differences between species. Intermediate portions of the GRN hierarchy are conserved at intermediate levels (this picture is further developed in Peter and Davidson 2011, 2015; cf. Wagner 2014). Thus, while anti-gradualism plays a crucial role in explaining deep conservation, it is part of a broader explanatory strategy for explaining why some parts of the genome evolve more readily than others.

\section{Two Paths to Anti-Gradualism}

Given that contemporary evo-devo explains deep conservation through a localized form of antigradualism, and that it invokes these deeply conserved features in causal explanations of evolutionary change, we may ask whether this anti-gradualism is sui generis or whether it rests on any broader patterns of thought. Here, I distinguish two bases of anti-gradualism: natural state thinking and Cuvierian functionalism. This analysis is meant to replace Mayr's sweeping unification of all forms of anti-gradualism with a piecemeal approach that nonetheless allows us to recognize shared patterns of thought in temporally separated biologists. I stress that the two patterns I discuss are not exhaustive: neither, for instance, fits the arch-saltationists Richard Goldschmidt and Otto Schindewolf. I select them because they are useful for understanding contemporary evo-devo: what it is, what it is not, and how it fits together with mainstream evolutionary theorizing. 


\subsection{Natural state thinking}

Elliott Sober, in an attempt to make Mayr's diatribes against "typological thinking" and "essentialism" philosophically precise, identified a type of model that he called a natural state model. On such a model, one distinguishes "between the natural state of a kind of object" and those non-natural states "produced by subjecting the object to an interfering force" (Sober 1980, 360). Evolutionary versions of natural state models treat species as having natural states around which individuals vary. Imposing selection on a population of such individuals can draw the population away from its natural state, but, once the selection is removed, the population will revert to the natural state. Moreover, there is only so far that selection can modify the population away from its natural state: variation is constrained.

In the nineteenth century, two important phenomena appeared to support relying on natural state thinking to understand how species could and could not change (cf. Lyell 1832, vol. 2, chaps. 1-4). First, it was well-known that one could rapidly produce large changes in domesticated species, but that such efforts could only proceed so far before hitting limits. Second, it was equally well-known that, as soon as such selection was relaxed, domesticated species showed a marked tendency to revert to their wild forms. Together, these phenomena suggested that artificial selection was best understood as an interfering force that could modify species only to a limited extent.

Natural state thinking played an important role in early opposition to Darwin, as exemplified by Fleeming Jenkin's (1867) review of Darwin's Origin. While Jenkin raised a diverse set of objections to Darwin's theory, Jenkin's argument that natural selection could not produce new species by accumulating small variations rested on his endorsement of a natural state model.

Jenkin argued that natural selection cannot accumulate small variations indefinitely because, as the experience of breeders shows, such accumulation soon hits limits beyond which it cannot proceed further. While great variability may exist at first, the rate at which new variants in a given direction arise "is a constantly diminishing rate, tending therefore to a limit" (Jenkin 1867, 281). Thus it appeared to Jenkin that there exist empirically determinable limits to the variability of species, and that these limits never exceed species boundaries. Species, Jenkin $(1867,282)$ argued, are contained "within a sphere of variation," with the average individual at the center. Individuals could vary in any direction, but were more likely to vary toward the center of the sphere. Artificial selection could push a population toward the edge of the sphere, but no further, and as soon as selection was relaxed, the population would start moving back toward the center. Importantly, this reversion was explained, not by the re-imposition of selection pressures in a direction contrary to the artificial selection regime, but in terms of the inherent variational properties of the species: variation predominantly trended toward the natural state. Species thus appeared to have an inherent tendency to stability, a tendency that the gradual accumulation of small variants could not overcome. Speciation, if it occurred at all, must occur by saltation. Jenkin $(1867,294)$ in fact allowed for such saltational evolution as a possibility, though he correctly noted that this "does not express the Darwinian theory."

I've described this example at some length in order to make apparent just how distinct it is from the explanation of deep conservation considered in the previous section. Natural state models have three key features that are not shared by explanations of deep conservation in terms of GRN kernels. First, natural state models are models of how variants can arise: variations can only depart so far from, and show a tendency to return to the natural state (Sober 1980, 350). GRN kernels do not restrict variation in this way, and thus GRN kernels should not be understood as natural states. Genetic variants can arise anywhere in a GRN kernel, in any direction, and show no internally driven tendency to revert back to the conserved state. Nothing 
in the nature of mutation itself favors the "natural" state, which is therefore not best conceived as a natural state at all.

Second, a natural state model predicts that modifications to the natural state can persist only so long as the interfering force is present: remove the interfering force and there will be a return to the natural state. On Sober's (1984) preferred interpretation of population genetics as a theory of forces, a mutation that disrupts a GRN kernel might at first glance be treated as an interfering force that pushes a population away from fixation for that kernel. However, in the absence of further forces (new mutations, migrations, drift, selection), the population will remain in Hardy-Weinberg equilibrium with that mutation present (Sober 1980, 380-81): there will be no reversion to the "natural" state. The persistence of GRN kernels requires the operation of strong selection that counteracts the effects of mutation. That a selective force is required to explain the persistence of the GRN kernel again shows that the kernel should not be understood as a natural state.

Third, natural state models, as a form of essentialism, specify features that any individual organism must have to count as a member of a species or higher group-groups must be able to be given what Sober calls "constituent definitions" (Sober 1980, 355). GRN kernels, however, cannot serve as properties mentioned in constituent definitions. While it may be true that all adult sea urchins possess the endomesoderm kernel, a sea urchin that lacks a gene in that kernel is nonetheless still a sea urchin (because its parents are sea urchins). It is just a sea urchin that cannot survive for very long. Moreover, while this non-viability prevents the accumulation of mutations affecting the kernel, there are no limits on how much a GRN kernel could vary while still belonging to an individual of the same species. In principle, the network topology of the endomesoderm kernel in sea urchins could change, linkage by linkage, until none of the original linkages remain, and yet the resulting population would still be a population of sea urchins. Kernels are not essences.

If, therefore, we are to find a general pattern of thought underlying this particular explanation of deep conservation, we must look elsewhere than natural state thinking. This in no way undermines Sober's analysis of the role of natural state models in underwriting anti-gradualism. It just shows that Mayr's hope to find a single basis for all opposition to gradualism cannot succeed. There are multiple paths to anti-gradualism.

\subsection{Cuvierian functionalism}

The work of the nineteenth century comparative anatomist Georges Cuvier illustrates a second path to anti-gradualism. Though Cuvier's explicit arguments against Lamarck consisted primarily of empirical evidence against the occurrence of transmutation (Rudwick 1997, 226-32) or else criticized Lamarck's theory for being overly speculative (Cuvier 1836), Cuvier's work furnishes a theoretical rationale for thinking that transmutation is not only does not occur but cannot occur. In reconstructing Cuvier's view, I draw both on Cuvier's own writings (Cuvier 1831, 1836; Rudwick 1997), as well as on secondary interpretations (Coleman 1964; Burkhardt 1977; Russell 1982; Outram 1986; Appel 1987). Coleman's account is especially interesting, since he explicitly argues that Cuvier was "the high priest of typology" and that this underwrote his opposition to transmutation (Coleman 1964, 98), and yet his own excavation of Cuvier's reasoning reveals a quite different basis for Cuvier's arguments.

At the heart of Cuvier's thought was the principle of the "conditions of existence." This principle began from the truism that viable organisms must be constructed in such a way that they can survive in the environment(s) in which they are found. Cuvier wrote: "Every organized being forms a whole, a unique and closed system, in which all the parts correspond mutually, 
and contribute to the same definitive action by a reciprocal reaction" (Rudwick 1997, 217). This principle played a central role in Cuvier's comparative anatomical work: it was on this basis that he claimed that, from a single part, "up to a certain point one can infer the ensemble" (Rudwick $1997,50)$, that is, the entire organism.

For Cuvier, this notion had both an internal and an external face: an organism must be integrated both "with regard to itself" and with regard "to its surrounding relations" (Cuvier 1831, 4; cf. Coleman 1964, 68; Burkhardt 1977, 194; Appel 1987, 46). Internally, the parts of an organism must be functionally integrated: they must work together to form a coherent whole. Externally, the particular form this functional integration takes must be suited to a particular environment.

Russell $(1982,34)$ has argued that the internal face is the more important of the two in $\mathrm{Cu}^{-}$ vier's thought, and that "the idea of the external conditions of existence, the environment, enters very little into this thought" (cf. Outram 1986, 348, who notes that Cuvier's teleology concerned "the inner economy of the organism"). While the claim that the environment played little role in Cuvier's thought is too strong, there is an important sense in which Cuvier stressed internal functional integration. The reason for this can be seen if we think about Cuvier's application of the principle of conditions of existence to classification: each taxonomic grouping was characterized by a particular, functionally determined plan of integration. Since taxonomic groups form a nested hierarchy, Cuvier could distinguish between "general conditions" (e.g., carnivory) and "particular conditions" (e.g., the features of specific prey items), which determined features shared at different ranks of the hierarchy (Rudwick 1997, 217).

Where particular conditions concern the specific environment of a given species, general conditions concern a larger range of environments, which can only be characterized abstractly. ${ }^{\mathrm{Q}} \mathrm{As}$ one moves up the taxonomic hierarchy, the connection between conditions of existence and particular environments gets weaker and weaker. Thus, for instance, Cuvier believed there existed a basic vertebrate plan of integration, determined by functional requirements, that all vertebrates shared, and that separated them from, e.g., mollusks, which were built on a different plan of integration (Appel 1987, 45; cf. Cuvier 1831, 6). However, there is no particular environment common to all vertebrates. From 1812 on, Cuvier based the division of animals into separate embranchements on the organization of their nervous system, which was involved with functions concerning perception, voluntary movement, and sensibility (Outram 1986, 324-27) - that is, relations to the environment in general, but not any particular environment (see Cuvier 1831, 11-13 for examples of environment-independent explanations of functional integration). At the opposite extreme, Cuvier argued that the variability within species was largely limited to "superficial" traits-those most closely tied to particular environmental conditions, such as the color and thickness of hair (Rudwick 1997, 226).

Thus, it was the emphasis on internal functional integration that lay at the root of Cuvier's important notions of the correlation and subordination of parts (Coleman 1964, 67). I shall therefore follow Russell $(1982,238-40)$ in treating emphasis on functional integration within the organism as the core of specifically Cuvierian functionalism, in contrast to Darwinian functionalism, which stresses adaptation to particular environments (more on this below, §6). Note that I am not claiming that these two forms of functionalism are incompatible, nor that either Cuvier and Darwin stressed one to the complete exclusion of the other. For now, I am merely claiming that they are importantly different.

\footnotetext{
${ }^{2}$ Outram (1986, 359-60) argues that Cuvier's notion of conditions of existence played a much larger practical role regarding higher groups than concerning lower groups; that is, it was most important precisely where "particular conditions" were least important.
} 
Cuvier's functionalism underwrote his arguments in favor of the view that every taxonomic group was a sharply defined, morphologically stable unit that was not susceptible to transmutation (Coleman 1964, 99; Appel 1987, 4, 41). In particular, he thought that this necessity for functional integration limited the range of viable variation. Central organs (e.g., the heart and nervous system) could not vary as much as less essential features (e.g., hair and color), as variations to these organs were more likely to upset the overall integration of the organism (Coleman 1964, 143). Furthermore, modification in any one part necessitated modifications in many others, again to preserve functional integration: "None of [an organized being's] parts can change without the others changing too" (Rudwick 1997, 217; cf. quotations in Outram 1986, 334, 357; Appel 1987, 51). Boundaries between taxonomic groups were sharply defined because intermediate forms, should they ever arise, would not be viable (Coleman 1964, 90; Burkhardt 1977, 193). Cuvier was explicit that functional considerations limits the range of possible forms: "Therefore there are only a certain number of possible combinations among the modifications which can be experienced by the principle organs, and beneath them there are only a certain number of possible subordinate combinations" (quoted in Outram 1986, 334; cf. Appel 1987, 45). The non-viability of intermediates equally rules out the possibility of gradual transformation, which would require proceeding through such intermediate stages.

The line of reasoning summarized above can be condensed into a three-step argument against the possibility of gradual evolution. First, organisms must be functionally integrated in order to survive (principle of the conditions of existence). Second, this need for functional integration renders intermediate forms non-viable, in virtue of lacking the requisite integration. Third, the non-viability of intermediate forms precludes the possibility of gradual transformation, since gradual transformation by definition must proceed through intermediate forms. Note that no metaphysical commitment to the underlying reality of types is presupposed here. It is not the invariability of types (i.e., group characters) that explains the impossibility of transmutation. Rather, the invariability of types and the impossibility of transmutation receive a common explanation in terms of the non-viability of intermediates.

It is also worth noting that this Cuvierian functionalist argument against the possibility of transmutation does not rest on beliefs about the nature of variation, in contrast to natural state thinking. Cuvierian functionalism says nothing about what variants can arise; it only concerns those variants that can survive. It rests entirely on the non-viability of intermediates. It is because the intermediates are non-viable, rather than because they cannot arise, that Cuvierian functionalism precludes gradual evolution.

Cuvierian functionalism therefore offers a path to anti-gradualism that departs quite radically from Mayr's account of typological thinking, despite Coleman's $(1964,98)$ diagnosis of Cuvier as "the high priest of typology." Mayr's notion of typological thinking implied a close connection between anti-gradualism and the way in which one understands variation. This connection is maintained in Sober's account of natural state thinking, but is lost in my reconstruction of Cuvierian functionalism. ${ }^{3}$

We can therefore see that Cuvier's three-step argument against transmutation, based on his functionalist commitments, provides a second path to anti-gradualism, distinct from the path

\footnotetext{
${ }^{3}$ Note that I am not claiming that Cuvierian functionalism and natural state thinking are incompatible, merely that Cuverian functionalist explanations do not require accepting a natural state model. The compatibility of the two is exemplified historically by St. George Mivart's On the Genesis of Species. In that work, he both reiterated Jenkin's arguments based on natural state thinking (Mivart 1871,118) and challenged the ability of Darwin's theory to account for "the infinitesimal commencement of structures" on Cuvierian functionalist grounds (Mivart 1871, $23,52)$.
} 
relying on natural state thinking. In the next section, we will see that it plays an important role in explanations of deep conservation that have emerged from evo-devo.

\section{Cuvierian Functionalism in Evo-Devo}

The explanation of deep conservation presented above $(\$ 3)$ repeats Cuvier's three-step argument point-for-point, albeit in a quite different argumentative context. In particular, where Cuvier applied his functionalist argument globally, considering the functional integration of the entire organism, Davidson and Erwin apply the argument in locally restricted settings: they apply it to particular GRN subcircuits. In their discussion of these subcircuits, they recapitulate each of the three steps of Cuvier's argument.

Davidson and Erwin's argument begins by noting the high degree of functional integration of GRN kernels. Kernels are integrated in two ways. First, they are recursively wired, meaning that they are characterized by extensive positive feedback. This allows them to play a crucial developmental role: they can convert transient inputs into a stably maintained regulatory state (Wagner 2014, chap. 3; Peter and Davidson 2015, chap. 6). Moreover, because of this recursive wiring, the expression of each gene in the kernel requires the proper functioning of multiple other genes in the kernel, meaning that the loss of a single gene can destroy kernel function entirely (Davidson and Erwin 2006).

Second, kernels are integrated into the total developmental process of the organism, i.e., they are highly generatively entrenched (Wimsatt 2015). Numerous developmental events are downstream of the development of endomesoderm in echinoderms. Disrupting the endomesoderm kernel leads to the failure to develop endomesoderm, which in turns leads to the failure of these downstream processes. The result is a dead embryo: Mutations that disrupt the endomesoderm kernel are lethal. This leads into the second step of the argument: Intermediate forms, i.e., those that involve small changes to kernel topology, are non-viable. And this explains why kernel topologies are deeply conserved-why, once they're in place, they simply fail to evolve (step three: anti-gradualism).

Note that both of these sorts of functional integration are inward-looking. The strong selection pressures that work to conserve kernel function are not imposed by particular features of the environment in which echinoderms find themselves, but by features of the organism that lead to kernel disruptions being invariably lethal in all naturally occurring environments. Thus, the inward-looking functional integration of GRN kernels leads to the impossibility (or at least extreme unlikeliness) of their evolving gradually.

A word is required about what it means for kernels to evolve gradually. Gradual change is most readily understood at the phenotypic level, where it implies that large phenotypic changes are produced by the continuous accumulation of smaller such changes. As all genetic changes are discontinuous, the notion of gradual evolution is effectively meaningless at the genetic level. Though changes to GRN structure are technically phenotypic changes, as they are the consequences of (rather than identical to) changes in DNA sequence, the best available models of GRN structure are nonetheless discrete. Either a gene actives/represses another, or it does not, and so a linkage is either present in a GRN, or it is absent (Peter and Davidson 2015, chap. 1). In this regard, GRN evolution, like genome evolution, is discontinuous, and the idea of gradual evolution does not straightforwardly apply. Provisionally, however, we might understand gradualism at the GRN level as involving the change of a single regulatory linkage at a time, in contrast to large-scale rewiring in a single step.

Understanding GRN-level gradualism in this way, we can expand on the sense in which Davidson and Erwin argue for the non-viability of intermediates as applied to kernels. The 
recursive wiring of kernels makes it difficult for them to evolve gradually, i.e., one linkage at a time, once they are established. Any one change would require compensatory changes to preserve the kernel's function. This is a GRN-level analog of Cuvier's view that single parts cannot change independently: change to any one part requires multiple coordinated changes. Since no mechanism is known that could simultaneous produce coordinated changes to multiple linkages, saltatory evolution of kernels is off the table. The only remaining option is non-evolution: once formed, kernels cease evolving.

As my aim in this paper is ultimately to show the important role that Cuvierian functionalism plays in evo-devo, I have focused on developmental genetic explanations of deep conservation. Now that the Cuvierian functionalist argument pattern is on the table, however, we can recognize that it is in fact applied rather widely. In particular, Wimsatt's (1986, 2013, 2015; Schank and Wimsatt 1986) notion of generative entrenchment captures a form of Cuvierian functionalist reasoning, and his work over the past three decades has shown its wide applicability. Generative entrenchment emphasizes diachronic dependencies within a "developmental trajectory" (Wimsatt 2015, 366): traits are generatively entrenched "to the degree that they have a number of later developing traits depending on them" (Wimsatt 1986, 198). The more downstream traits that depend on a given developmental event, the more likely it is that changes to that event will cause "cascading disruptions in development" (Wimsatt 2013, 25). Models of generative entrenchment thus provide one way of locally applying Cuvierian functionalist reasoning: they show that gradual evolution is difficult in highly entrenched parts of an organism, and thus explain why evolutionary change is displaced onto other parts of the organism.

However, the full scope of Cuvierian functionalist reasoning in biology remains to be determined. Cuvierian functionalism includes not only diachronic dependencies but also synchronic dependencies, which have been less thoroughly explored philosophically (but see Wouters 2005, 2007; Reiss 2009). For example, the recursive wiring of a GRN kernel is a case of such synchronic dependency of the parts (kernels are also generatively entrenched, as we have seen). Wagner's and Schwenk's (2000; Schwenk and Wagner 2001) work on evolutionary stable configurations furnishes another example of the importance of synchronic dependencies. Determining the prevalence of Cuvierian functionalist reasoning within the biological sciences remains a task for future work.

\section{Cuvierian Functionalism and Darwinian Functionalism}

Earlier (§4.2), I suggested that Cuvierian functionalism differs from Darwinian functionalism by focusing on internal functional integration of the organism, in contrast to Darwin's emphasis on the adaptation of organisms to particular environments. In this section, I expand this point into a fuller characterization of their differences, and use this as an opportunity to consider why it is that evo-devo is not and cannot be a purely Darwinian research program.

Darwinian functionalism and Cuvierian functionalism differ in four primary ways: their treatment of variation, whether they are inward-looking or outward-looking, their relationship to taxonomic levels, and their primary explanatory targets. I take up each in turn, under the headings Variation, Environment, Rank, and Explanandum.

Variation. Darwinian functionalism emphasizes the gradual accumulation of small variations under the influence of natural selection. In the context of the genotype-phenotype distinction, we can specify that "small" here refers to the size of the phenotypic effect of genetic mutations, not on the nature of the mutations themselves, considered as changes in DNA sequence, as it has long been recognized that the genetic "size" of a mutation is not closely correlated to its phenotypic size (Wright 1963). For Darwin, variation was widespread in nature,

○ OPEN ACCESS - PTPBIO.ORG 
furnishing resources for selection to gradually sculpt parts of organisms to better serve particular functions. Darwin's (1859, chap. 6) classic thought experiment about the evolution of the eye from a simple, light-sensitive nerve is a paradigm case of this.

As noted above (\$4.2), Cuvierian functionalism is not primarily a claim about the nature of variation. In the context of GRN kernels, for instance, Cuvierian functionalist reasoning does not involve claiming that they vary in ways different from other features of the organism. Rather, it is assumed that the regions of the genome on which kernel topology is based are just as susceptible to mutation as any other region of the genome. However, Cuvierian functionalism does differ from Darwinian functionalism in its treatment of variation in this respect: because variants are non-viable, there are no prospects for accumulation. Thus, the creative aspect of natural selection, so crucial to Darwinian functionalism (Godfrey-Smith 2009, 49-51), is absent from Cuvierian functionalism.

Environment. Darwinian functionalism is outward-looking. Selection pressures are generated by relations between organisms and their environment. What is beneficial in one environment may be harmful (or neutral) in another environment, as exemplified by the nonfunctionality of the eyes of many cave-dwelling species, which occupy dark environments in which light-sensitive organs are useless. As environments change, the selection pressures that affect the evolution of a population also change. Cuvierian functionalism, by contrast, is inwardlooking. Selection pressures are generated by the internal functional integration of the organism and thus are relatively environment-independent.

Recognizing this allows us to better understand the relationship between Darwinian functionalism and Cuvierian functionalism. Rather than erect a dichotomy between environmentdependent selection pressures and environment-independent selection pressures, we should recognize the existence of a continuum of environment-sensitivity of selection pressures. Cuvierian functionalism emphasizes selection pressures that are highly environment-insensitive, but they need not be absolutely so. It is at least conceivable, for instance, that an ingenious scientist could develop a laboratory environment in which a sea urchin with a non-functional endomesoderm kernel could nonetheless survive (e.g., by injecting the right downstream gene products into the right cells at the right times). Probably no mutation is lethal in an entirely environmentinsensitive manner. Still, the selection pressures acting on mutations that disrupt the endomesoderm kernel are more environment-insensitive than, e.g., those that affect eye function.

The deployment of Cuvierian functionalist reasoning in evo-devo rests on a recognition of this point. Thus, for instance, Eric Davidson's arguments about the evolution of GRNs recognizes that different kinds of GRN subcircuits show variable degrees of conservation (Davidson and Erwin 2006; Peter and Davidson 2011). Kernels merely represent one extreme. Günter Wagner (2014) makes a similar point in his discussion of GRNs and homology, noting that many GRN subcircuits that share kernels' property of being recursively wired (and so are subject to Cuvierian functionalist constraints) are less generatively entrenched than kernels. Thus they are more evolutionarily labile than kernels, though not as labile as less tightly integrated features.

Rank. Darwinian functionalism is population-specific. Populations, once gene flow is cut off, experience independent evolutionary trajectories. As Ron Amundson $(2005,256)$ puts the point, "once speciation occurs, no causal force can unify distinct populations." That is, without gene flow, distinct populations will experience mutations and selection pressures independently. 
Mutations will arise and spread (or fail to spread) through these populations independently. (1 $^{-}$ Once gene flow is cut off, populations no longer share their evolutionary fate.

Cuvierian functionalism, by contrast, transcends populations, and can be applied at all ranks of the taxonomic hierarchy. This can be seen in Cuvier's own work: he believed that plans of functional integration existed at the level of his four embranchements as much as at the level of particular species. Thus, there was for Cuvier a vertebrate plan of integration, distinct from the molluscan plan, just as much as there was a zebra plan of integration, distinct from the horse plan. The same holds for contemporary Cuvierian functionalists: We have just seen how Davidson and Wagner recognize the existence of degrees of integration that coincide with degrees of conservation. There is integration common to Chordata and Arthropoda (the heart kernel); there is also integration common to frogs (the generalized frog foot). Note that, if the population-specificity of Darwinian functionalism is interpreted as forbidding reference to any features above the species level in evolutionary explanations (Amundson 2005, chap. 11), then there is a conflict between Darwinian and Cuvierian functionalism. This issue is discussed below (§7).

Explanandum. Finally, Darwinian functionalism and Cuvierian functionalism differ in their target explananda. The primary explanandum of Darwinian functionalism is evolutionary change (and the production of organismic diversity via evolutionary change), in particular adaptation to shifting environments. Because selection pressures are environment-specific, as environments change, new selection pressures are generated (stasis can thus be explained insofar as environments remain constant). The emphasis on change makes sense in light of the context in which Darwin developed his theory. At that time, heredity was understand as a force that promoted stasis, and thus it was evolutionary change that specifically required explaining (Gayon 1998). This understanding of heredity was abandoned by the Mendelians (Gayon 1998), but change and diversity remained the primary target of Darwinian functionalist explanations (Dobzhansky 1982, chap. 1).

In fact, precisely the features that make Darwinian functionalism so good at explaining change-its emphasis on the widespread availability of variation, its emphasis on environmentally generated selection pressures, and its population-specificity-make it rather difficult to explain stasis within a purely Darwinian functionalist framework. This problem was raised early in the history of evo-devo, in a classic paper titled "On the Problem of Stasis in Organismal Evolution" (Wake, Roth, and Wake 1983). They write:

Perhaps no phenomenon is as challenging to evolutionary biologists as what has been termed "stasis". With natural selection operating in a changing environment as an agent of adaptation, we expect to see changes at the organismal, ultimately physiological and morphological, level. How, though, can we explain the paradoxical situation in which environments change, even dramatically, but organisms do not? (Wake, Roth, and Wake 1983, 212, in-text citation removed)

More recently, Olivier Rieppel has made a similar point:

Stable developmental stages preserved through geologic time create a puzzle for Darwinian evolutionary theory, based as it is on fundamental variation, natural selection, and continuity of transformation. (Rieppel 2010, 668)

\footnotetext{
${ }^{4}$ Of course, if the two populations interact in ways other than interbreeding, e.g., via predation or competition for resources, then they can impose new selection pressures on one another. The point, however, still holds: changes to the genetic composition of one population cannot spread to the other population.
} 
The basic puzzle is simple. If small variants are produced for all parts of the organism, and if natural selection accumulates these variants over time, and if these selection pressures are generated by features of the particular environment in which a population finds itself, and if divergent populations experience independent evolutionary trajectories, how can anything remain constant over long periods of time (during which environments change dramatically) and in multiple distinct lineages (which occupy a large range of niches at any given time)? How can we explain the stability of animal body plans across environments and populations in terms of selection pressures that are relative to particular environments and populations?

As we have seen $(\$ 3)$, the deep conservation of animal body plans is one of the central explanatory concerns of evo-devo. For this reason, evo-devo cannot be a purely Darwinian functionalist research program. This is not to say that evo-devo cannot employ Darwinian functionalist reasoning (cf. Breuker, Debat, and Klingenberg 2006). It simply means that, when it comes to stasis, something else is required. That something else is Cuvierian functionalism, whose central explanatory aim is stasis. Precisely because functional integration can generate environment-insensitive selection pressures, it can explain why, once such functional integration arises, it is preserved both within lineages as environments shift and across lineages as they come to occupy different niches.

Thus, despite their differences, Cuvierian functionalism and Darwinian functionalism are complementary. The history of life on earth involves both dramatic change and equally dramatic stasis. Evolutionary theory must be able to accommodate both. Supplementing Darwinian functionalism with Cuvierian functionalism gives it the resources to do so.

\section{Cuvierian Functionalism and Exclusive Population Thinking}

At the end of the previous section, I presented a rosy picture of Darwinian functionalism and Cuvierian functionalism working together to explain the gamut of evolutionary phenomena. I believe this picture is largely if imperfectly realized in the current state of biological science. However, there is an issue that threatens it. This lies in Darwinian evolutionary theory's alleged commitment to what Ron Amundson (2005, chap. 11) calls "exclusive population thinking." My aim in this section is to defuse this issue by showing that the population-specificity of Darwinian functionalism does not require commitment to exclusive population thinking (indeed, such commitment would rule out far more of evolutionary biology than Amundson realizes). Once the demands of population thinking are properly understood, Cuvierian functionalism can be shown to meet these demands. I will also briefly discuss the implications this has for relating Cuvierian functionalism to structuralist reasoning in biology.

Throughout this paper, I've been making the case that understanding the role of Cuvierian functionalism in evo-devo can replace analyses of evo-devo as typological. In that regard, it might be thought that my analysis ipso facto eliminates any conflict between evo-devo and population thinking. Nonetheless, Cuvierian functionalism does preserve a key feature of typological thinking: causal explanations of evolutionary change that refer to taxonomic ranks higher than the species. By offering a unified explanation of phylum-level conservation, Cuvierian functionalist reasoning offers evolutionary explanations in terms of constraints that affect divergent lineages undergoing independent evolutionary trajectories. This conflicts with what Amundson calls "exclusive population thinking."

Amundson offers two distinct characterizations of exclusive population thinking. The first is ontological: "Once speciation occurs, no causal force can unify distinct populations" (Amundson 2005,256$)$. The point is the same as that made above (\$6) about the population-specificity of Darwinian functionalism. Interbreeding populations evolve as a unit, experiencing a particu- 
lar set of selection pressures generated by a particular environment and acting on mutations that will spread or fail to spread throughout the entire population. Even in cases with complex population structure (e.g., multiple sub-populations that experience gene flow but occupy different environments and so experience different selection pressures), mutations that arise and spread in one sub-population can, in virtue of gene flow, spread to the other sub-populations (Pressoir and Berthaud 2004). Their evolutionary histories are yoked together for as long as gene flow remains.

Once gene flow is cut off, however, populations will experience distinct sets of mutations as well as distinct selection pressures. Without gene flow, the spread of mutation to a different population is impossible, so the evolutionary trajectories of distinct populations are split. It is true that, if the two populations interact, the spread of a new mutation in one population $\left(P_{1}\right)$ can affect the evolution of the other $\left(P_{2}\right)$, but only in virtue of generating a new selection pressure that acts on $P_{2}$. Even though the populations are causally interacting, they are not causally unified in the relevant sense. On this ontological reading of exclusive population thinking, the claim is true. Gene flow is the only evolutionary force that can unify evolutionary trajectories in the relevant sense.

However, Amundson $(2005,238)$ also offers a second, epistemological characterization of exclusive population thinking "as the position that no generalizations or concepts that refer to higher taxonomic levels are relevant to the causal understanding of evolutionary change." Whereas the first characterization concerns the causal importance of gene flow in binding together populations, the second characterization concerns the structure of our evolutionary explanations. Cuvierian functionalist explanations, which invoke functional constraints at all levels of the taxonomic hierarchy, patently violate exclusive population thinking in this sense. Thus, if there is good reason to be an exclusive population thinker in this second sense, then there's a problem for evo-devo.

At this point, however, we may ask two related questions: (1) Is Darwinian evolutionary theory really committed to exclusive population thinking in its epistemological sense? and (2) Does the epistemological version of exclusive population thinking follow from the ontological? I will argue for a negative answer to both questions. Since it is the epistemological interpretation that primarily concerns patterns of thinking, and since, as we will see, only the epistemological interpretation generates exclusivity (in the sense of forbidding Cuvierian functionalist patterns of thinking), henceforth I will use "exclusive population thinking" to refer specifically to the epistemological interpretation.

Regarding the first question, Darwinian evolutionary theory is not committed to exclusive population thinking. Evolutionary explanations offered outside of evo-devo routinely violate the epistemological strictures of exclusive population thinking. This can be seen by considering how evolutionary biologists explain ecological rules. Why, for instance, do many bird and mammal lineages tend to be larger in cooler climates (Bergmann's rule; Ashton, Tracy, and de Queiroz 2000; Meiri and Dayan 2003)? And why does this trend fail to occur in other lineages, such as amphibians and insects (Adams and Church 2008; Shelomi 2012)? If explanations that transcend single populations are forbidden, then each instance of this trend must be explained independently. It's reasonable to ask, however, for a more general explanation, one in terms of shared features of the groups that experience the trend leading those groups to experience similar selection pressures in similar environments, and thus to evolve convergently. In the case of Bergmann's rule, one proposed explanation invokes the need to minimize heat loss in colder environments (Walters and Hassall 2006), though this explanation has been challenged (Ashton, Tracy, and de Queiroz 2000). 
Even if this particular explanation is false, however, the key point is that the explanation has an acceptable form, despite unifying distinct populations. Even though the lineages experience these causes independently, we can nonetheless offer a unified explanation of their evolutionary trajectory. We can do so because the same causal factor is repeated in these lineages and affects them similarly.

We thus have an answer to our second question as well: Accepting that only gene flow can unify the evolutionary trajectories of distinct populations does not require abandoning explanations that refer to higher taxonomic ranks. Unified evolutionary explanations, in other words, do not require unified evolutionary causes; it is enough for similar causes to affect different populations independently. We are left, then, with no reason to endorse exclusive population thinking, on grounds independent of any considerations regarding evo-devo specifically.

Once we see this, we can see that Cuvierian functionalist explanations refer to higher taxonomic levels in a way that is justified in just the same way that explanations of ecological rules are justified. According to Davidson and Erwin, what underlies conserved body plan elements are conserved GRN kernels. These are conserved because changes to their structure are rarely if ever viable. Because of this, kernels are passed down to all of the descendants of the lineage in which they first arose, and thus are present in all populations in that lineage. Because they generate selection pressures in an environment-insensitive way, all populations in the lineage experience the same strong selection pressures for kernel preservation. No cause that unifies the separated populations is required. They are undergoing separate evolutionary trajectories. It's just that these separate trajectories are subject to the same constraints on kernel evolution.

This dissolution of the conflict between Cuvierian functionalism and (no longer "exclusive") population thinking closely resembles that offered by Tim Lewens (2009) in his "exculpation" of typological thinking. Lewens, too, offers an explanation in terms of the repetition of shared features in shared lineages giving rise to similar constraints-in this spirit he speaks of "nominalist types" (Lewens 2009, sec. 7). Importantly, however, Lewens is concerned with structuralist constraints (those that affect the probability of certain variants arising), rather than with $\mathrm{Cu}^{-}$ vierian functionalist constraints (which affect the probability that variants will be favored by selection) (Lewens 2009, sec. 8; cf. Amundson 1994). Earlier (\$3), I discussed two reasons why evo-devo might be thought typological: its emphasis on stasis, and its invocation of developmental types to explain change. The example I gave of an explanation in terms of developmental types (Alberch and Gale's model of the generalized frog foot) is a structuralist explanation in this sense.

Lewens's position and mine are therefore compatible. My exculpation of Cuvierian functionalist explanations covers one "typological" aspect of evo-devo, while Lewens's exculpation of developmental type explanations covers the other. However, Lewens treats these as two separate issues. I want to end this section by arguing that there is in fact a close connection between them. In particular, it is often precisely because Cuvierian functionalist constraints are in operation that developmental type explanations have the force they do.

Developmental type explanations explain evolutionary trajectories in terms of how the developmental system of an organism constrains the kinds of variants that can be produced. Thus, for instance, Alberch and Gale (1985) explain why frog lineages, if they lose any phalanges at all, always lose phalanges from the first toe by showing that the frog limb develops in such a way that any perturbation that leads to loss of any phalanges leads to loss of phalanges from the first digit. This constraint rests on features of limb development shared by all frogs. These features, however, can change, as evidenced by the fact that salamanders show a different pattern of digit loss (Alberch and Gale 1985). 
There are thus two explanatory tasks here: (i) to explain why the frog limb has the specific variational properties it has, and (ii) to explain why the features of frog limb development that generate those variational properties are conserved. Developmental type explanations address only the first task. When it comes to the second task, it should be clear that there is, at least potentially, a role to be played by Cuvierian functionalist argumentation. It may very well be that mutations that substantially change the variational properties of some developmental process are disruptive of functional integration and only selectively beneficial (or neutral) in rare circumstances (see Wagner 2014, especially the discussion of "variational modalities"). As Wallace Arthur $(2001,276)$ puts the point, "negative internal selection at one developmental stage will appear as constraint at the next." In my lingo: Cuvierian functionalist constraints can be precisely what generates Lewens's structuralist constraints.

Amundson (2005, chap. 11) concluded his discussion of exclusive population thinking by offering a choice: to harmonize evo-devo with the rest of evolutionary theory, either (i) evodevo must give up its reliance on explanations that rely on generalizations above the species level, or (ii) Darwinian evolutionary theory must give up its adherence to exclusive population thinking. We have seen that this choice is in fact a rather easy one, since nothing in evolutionary theory requires commitment to exclusive population thinking. Both Cuvierian functionalist explanations and the developmental type explanations they enable are safe.

\section{Universality and Diversity Revisited}

In this paper, I have attempted to elucidate the role of Cuvierian functionalist arguments in contemporary evo-devo, and to show how these arguments can be integrated into evolutionary theorizing without falling afoul of classic objections to "typological" thinking. In doing so, I have defended the following claims:

- There exist at least two distinct paths to anti-gradualism-Cuvierian functionalism and natural state thinking. These paths cannot be unified under any more general heading, such as Mayr's notion of "typological thinking”. (\$4)

- Evo-devo draws on localized applications of Cuvierian functionalist reasoning to explain deep conservation. It does not draw on natural state thinking. (§§3-5)

- Cuvierian functionalism is distinct from, yet compatible with and complementary to, Darwinian functionalism. Rather than treating the distinction between them as a dichotomy, these two forms of functionalism should be understood as extremes of a continuum. (\$6)

- Cuvierian functionalist reasoning in evo-devo is compatible with population thinking, properly understood. Interpretations of population thinking that exclude any role for Cuvierian functionalism are indefensible. $(\$ 7)$

In light of this, it is worth revisiting Raff's (1996, chap. 1) claim that evo-devo differs from neo-Darwinian evolutionary theorizing in emphasizing universality rather than diversity. This is often taken to support the view that evo-devo is a structuralist program in biology, in contrast to the functionalism of neo-Darwinian theorizing. This view structures Amundson's (2005) book-length treatment of the issue, and it has been endorsed by various evo-devo biologists (e.g., Alberch 1989; Wagner 2014). While a full treatment of the nature of structure-function disputes is a topic for another paper, structuralists can be roughly understood as those who see internal and/or ahistorical causal factors as the primary determinants of organismal form, 
while functionalists see external, historical causal factors (especially selection) as the primary determinants.

There is truth to this view-evo-devo has, from its inception up to the present day, had a strong structuralist strain (e.g., Alberch 1989; Kauffman 1993; Müller 2003; Newman 2012)_ but it's overly simplistic. If functionalism is conceived as including only Darwinian functionalism, with its emphasis on continuous variability and environment-driven selection pressures, and if structuralism is understood as the only alternative to functionalism, then evo-devo's emphasis on deep conservation can only seem structuralist. Alberch $(1989,25)$, for instance, seems to endorse something like this inference when he argues that functionalist approaches inherently treat nature as continuous.

But the inference is mistaken, because there's a third option: Cuvierian functionalism. Like Darwinian functionalism, Cuvierian functionalism (in its post-Darwinian forms) emphasizes the role of selection in determining organic form. Like structuralism, Cuvierian functionalism emphasizes what is universal (evolutionarily conserved) over what is diverse (evolutionarily labile), and does so by focusing on the internal organization of the organism, not its relation to any particular environment. Moreover, pace Alberch, it offers a functionalist explanation for the discontinuous nature in which actual organisms occupy morphospace. While it's true that evo-devo has always had a sizable structuralist contingent, evo-devo, especially those regions centered on developmental genetics, also has a strong Cuvierian functionalist contingent (e.g., Arthur 2001; Carroll 2008; Wagner 2014; Peter and Davidson 2015; Wimsatt 2015).

If we fail to recognize the role of Cuvierian functionalist reasoning in evo-devo, we will miss out on the internal complexity of evo-devo. Treating evo-devo as a monolithically structuralist research program that clashes with the functionalism of neo-Darwinian theorizing gets things wrong on both sides. As I've argued here, it leads to overstating the extent to which evo-devo conflicts with other forms of evolutionary theorizing. But it also gets evo-devo itself wrong, because it misses out on the structure-function disputes that arise within evo-devo. Stuart Newman and Ramray Bhat $(2008,2)$, for instance, criticize Sean Carroll's functionalist approach to evo-devo as "Neo-Neo-Darwinism." Understanding such disputes is crucial to developing a proper philosophical understanding of the conceptual foundations of evo-devo.

In order, therefore, to understand both the internal structure of evo-devo and the place of evo-devo within the broader landscape of evolutionary theorizing, we must recognize Cuvierian functionalism as an alternative to both structuralism and Darwinian functionalism.

\section{Acknowledgments}

I am grateful to James Lennox, Mark Wilson, Sandra Mitchell, Mark Rebeiz, James Woodward, Ford Doolittle, Jeremy Wideman, David Colaço, Nora Boyd, Bill Wimsatt, John Reiss, and two anonymous reviewers for helpful comments on earlier drafts of this material. I am also grateful to audiences at PSA 2018 and at Dalhousie University for helpful comments and discussion.

\section{Literature cited}

Adams, Dean C., and James O. Church. 2008. "Amphibians Do Not Follow Bergmann's Rule.” Evolution 62 (2): 413-20. https://doi.org/10.1111/j.1558-5646.2007.00297.x.

Alberch, Pere. 1989. "The Logic of Monsters: Evidence for Internal Constraint in Development and Evolution." Geobios 22 (January): 21-57. https://doi.org/10.1016/S0016-6995(89)80006-3. 
Alberch, Pere, and Emily A. Gale. 1985. "A Developmental Analysis of an Evolutionary Trend: Digital Reduction in Amphibians." Evolution 39 (1): 8-23.

Amundson, Ron. 1994. "Two Concepts of Constraint: Adaptationism and the Challenge from Developmental Biology.” Philosophy of Science 61 (4): 556-78. https://doi.org/10.1086/289822.

—. 1998. "Typology Reconsidered: Two Doctrines on the History of Evolutionary Biology." Biology and Philosophy 13 (2): 153-77. https://doi.org/10.1023/A:1006599002775.

- 2005. The Changing Role of the Embryo in Evolutionary Thought: Roots of Evo-Devo. Cambridge: Cambridge University Press.

Appel, Toby A. 1987. The Cuvier-Geoffroy Debate: French Biology in the Decades before Darwin. Oxford: Oxford University Press.

Arthur, Wallace. 2001. "Developmental Drive: An Important Determinant of the Direction of Phenotypic Evolution." Evolution and Development 3 (4): 271-78. https://doi.org/10.1046/j.1525-142x. 2001.003004271.x.

Ashton, Kyle G., Mark C. Tracy, and Alan de Queiroz. 2000. "Is Bergmann's Rule Valid for Mammals?" The American Naturalist 156 (4): 390-415.

Breuker, Casper J., Vincent Debat, and Christian Peter Klingenberg. 2006. "Functional Evo-Devo." Trends in Ecology E' Evolution 21 (9): 488-92. https://doi.org/10.1016/j.tree.2006.06.003.

Brigandt, Ingo. 2007. "Typology Now: Homology and Developmental Constraints Explain Evolvability." Biology and Philosophy 22 (5): 709-25. https://doi.org/10.1007/s10539-007-9089-3.

Burkhardt, Richard W. 1977. The Spirit of System: Lamarck and Evolutionary Biology. Cambride, MA: Harvard University Press.

Carroll, Sean B. 2008. "Evo-Devo and an Expanding Evolutionary Synthesis: A Genetic Theory of Morphological Evolution.” Cell 134 (1): 25-36. https://doi.org/10.1016/j.cell.2008.06.030.

Coleman, William. 1964. Georges Cuvier, Zoologist: A Study in the History of Evolution Theory. Cambridge, MA: Harvard University Press.

Coyne, Jerry A. 2006. "Comment on 'Gene Regulatory Networks and the Evolution of Animal Body Plans.”' Science 313 (5788): 761b-761b. https://doi.org/10.1126/science.1126454.

Cuvier, Georges. 1831. The Animal Kingdom Arranged in Conformity with Its Organization. Translated by H. M'Murtrie. 2nd ed. New York: G. \& C. \& H. Carvill.

_. 1836. "Biographical Memoir of M. de Lamarck." Edinburgh Nerw Philosophical Journal 20: $1-22$.

Darwin, Charles. 1859. On the Origin of Species by Means of Natural Selection, or the Preservation of Favoured Races in the Struggle for Life. London: John Murray.

Davidson, Eric H., and Douglas H. Erwin. 2006. "Gene Regulatory Networks and the Evolution of Animal Body Plans.” Science 311 (5762): 796-800. https://doi.org/10.1126/science.1113832.

Dobzhansky, Theodosius. 1982. Genetics and the Origin of Species. 1st ed. New York: Columbia University Press.Erwin,

Douglas H. 2015. "Was the Ediacaran-Cambrian Radiation a Unique Evolutionary Event?" Paleobiology 41 (01): 1-15. https://doi.org/10.1017/pab.2014.2.

Erwin, Douglas H., and Eric H. Davidson. 2006. "Response to Comment on 'Gene Regulatory Networks and the Evolution of Animal Body Plans.'” Science 313 (5788): 761c. https://doi.org/10. $1126 /$ science. 1126765 .

Erwin, Douglas H., and James W. Valentine. 2013. The Cambrian Explosion: The Construction of Animal Biodiversity. W. H. Freeman. 
Gayon, Jean. 1998. Darwinism's Struggle for Survival: Heredity and the Hypothesis of Natural Selection. Cambridge: Cambridge University Press.

Godfrey-Smith, Peter. 2009. Darwinian Populations and Natural Selection. Oxford: Oxford University Press.

Goldschmidt, Richard. 1982. The Material Basis of Evolution. New Haven: Yale University Press.

Hall, Brian K. 1999. Evolutionary-Developmental Biology. 2nd ed. Dordrecht: Springer Netherlands.

Hull, David L. 1965a. "The Effect of Essentialism on Taxonomy-Two Thousand Years of Stasis (I)." British Journal for the Philosophy of Science 15 (60): 314-26. https://doi.org/10.1093/bjps/XV.60. 314.

- 1965b. "The Effect of Essentialism on Taxonomy-Two Thousand Years of Stasis (II)." The British Journal for the Philosophy of Science 16 (61): 1-18. https://doi.org/10.1093/bjps/XVI.61.1.

Jenkin, Fleeming. 1867. "[Review of] The Origin of Species." The North British Review 46 (June): 277-318.

Jenner, Ronald A. 2006. "Unburdening Evo-Devo: Ancestral Attractions, Model Organisms, and Basal Baloney." Development Genes and Evolution 216 (7-8): 385-94. https://doi.org/10.1007/ s00427-006-0084-5.

Kauffman, Stuart A. 1993. The Origins of Order: Self-Organization and Selection in Evolution. Oxford: Oxford University Press.

Levin, Michal, Leon Anavy, Alison G. Cole, Eitan Winter, Natalia Mostov, Sally Khair, Naftalie Senderovich, et al. 2016. "The Mid-Developmental Transition and the Evolution of Animal Body Plans.” Nature 531 (7596): 637-41. https://doi.org/10.1038/nature16994.

Lewens, Tim. 2009. “What Is Wrong with Typological Thinking?” Philosophy of Science 76 (3): 35571. https://doi.org/10.1086/649810.

Love, Alan C. 2009. "Typology Reconfigured: From the Metaphysics of Essentialism to the Epistemology of Representation." Acta Biotheoretica 57 (1-2): 51-75. https://doi.org/10.1007/ s10441-008-9059-4.

Lyell, Charles. 1832. Principles of Geology; Being an Attempt to Explain the Former Changes of the Earth's Surface, by Reference to Causes Now in Operation. Vol. 2. London: John Murray.

Mayr, Ernst. 1959. "Darwin and the Evolutionary Theory in Biology." In Evolution and Anthropology: A Centennial Appraisal, 1-10. Washington, D.C.: The Anthropological Society of Washington.

- 1960. "The Emergence of Evolutionary Novelties." In Evolution after Darwin, Vol. 1, The Evolution of Life: Its Origin, History, and Future, edited by Sol Tax, 349-80. Chicago: University of Chicago Press.

Meiri, Shai, and Tamar Dayan. 2003. “On the Validity of Bergmann's Rule.” Journal of Biogeography 30 (3): 331-51. https://doi.org/10.1046/j.1365-2699.2003.00837.x.

Mivart, St. George. 1871. On the Genesis of Species. London: MacMillan and Co.Müller, Gerd B. 2003. "Homology: The Evolution of Morphological Organization." In Origination of Organismal Form: Beyond the Gene in Developmental and Evolutionary Biology, edited by Gerd B. Müller and Stuart A. Newman, 51-69. Cambridge, MA: MIT Press.

Newman, Stuart A. 2012. "Physico-Genetic Determinants in the Evolution of Development." Science 338: $217-20$.

Newman, Stuart A., and Ramray Bhat. 2008. "Dynamical Patterning Modules: Physico-Genetic Determinants of Morphological Development and Evolution.” Physical Biology 5 (1): 015008. https://doi.org/10.1088/1478-3975/5/1/015008. 
Outram, Dorinda. 1986. "Uncertain Legislator: Georges Cuvier's Laws of Nature in Their Intellectual Context." Journal of the History of Biology 19 (3): 323-68. https://doi.org/10.1007/BF00138285.

Peter, Isabelle S., and Eric H. Davidson. 2011. "Evolution of Gene Regulatory Networks Controlling Body Plan Development." Cell 144 (6): 970-85. https://doi.org/10.1016/j.cell.2011.02.017.

- 2015. Genomic Control Process: Development and Evolution. Saint Louis: Academic Press.

Pressoir, G., and J. Berthaud. 2004. "Population Structure and Strong Divergent Selection Shape Phenotypic Diversification in Maize Landraces.” Heredity 92 (2): 95-101. https://doi.org/10.1038/sj. hdy.6800388.

Raff, Rudolf A. 1996. The Shape of Life: Genes, Development, and the Evolution of Animal Form. Chicago: University of Chicago Press.

Reiss, John O. 2009. Not By Design: Retiring Darwin's Watchmaker. Berkeley: University of California Press.

Rieppel, Olivier. 2010. "New Essentialism in Biology." Philosophy of Science 77 (5): 662-73. https: 7/doi.org/10.1086/656539.

Rudwick, Martin J. S. 1997. Georges Cuvier, Fossil Bones, and Geological Catastrophes: Nerw Translations $E$ Interpretations of the Primary Texts. Chicago: University of Chicago Press.

Russell, Edward Stuart. 1982. Form and Function: A Contribution to the History of Animal Morphology. Chicago: University of Chicago Press.

Schank, Jeffrey C., and William C. Wimsatt. 1986. "Generative Entrenchment and Evolution." PSA: Proceedings of the Biennial Meeting of the Philosophy of Science Association 2: 33-60.

Schindewolf, Otto H. 1993. Basic Questions in Paleontology: Geologic Time, Organic Evolution, and Biological Systematics. Edited by Wolf-Ernst Reif. Translated by Judith Schaefer. Chicago: University of Chicago Press.

Schwenk, Kurt, and Günter P. Wagner. 2001. "Function and the Evolution of Phenotypic Stability: Connecting Pattern to Process." American Zoologist 41 (3): 552-63.

Shelomi, Matan. 2012. "Where Are We Now? Bergmann's Rule Sensu Lato in Insects." The American Naturalist 180 (4): 511-19. https://doi.org/10.1086/667595.

Sober, Elliott. 1980. "Evolution, Population Thinking, and Essentialism.” Philosophy of Science 47 (3): 350-83. https://doi.org/10.1017/CBO9780511624940.012.

- 1984. The Nature of Selection: Evolutionary Theory in Philosophical Focus. Chicago: University of Chicago Press.

Valentine, James W. 2004. On the Origin of Phyla. Chicago: University of Chicago Press.

Wagner, Günter P. 2014. Homology, Genes, and Evolutionary Innovation. Princeton: Princeton University Press.

Wagner, Günter P., and Kurt Schwenk. 2000. "Evolutionarily Stable Configurations: Functional Integration and the Evolution of Phenotypic Stability." In Evolutionary Biology, 31:155-217. Boston, MA: Springer US. https://doi.org/10.1007/978-1-4615-4185-1_4.

Wake, David B., Gerhard Roth, and Marvalee H. Wake. 1983. "On the Problem of Stasis in Organismal Evolution.” Journal of Theoretical Biology 101 (2): 211-24. https://doi.org/10.1016/ 0022-5193(83)90335-1.

Walters, Richard John, and Mark Hassall. 2006. "The Temperature冈Size Rule in Ectotherms: May a General Explanation Exist after All?” The American Naturalist 167 (4): 510-23. https://doi.org/ $10.1086 / 501029$. 
Wimsatt, William C. 1986. "Developmental Constraints, Generative Entrenchment, and the InnateAcquired Distinction.” In Integrating Scientific Disciplines, edited by William Bechtel, 185-208. Dordrecht: Martinus Nijhoff Publishers.

. 2013. "Evolution and the Stability of Functional Architectures." In Functions: Selection and Mechanisms, edited by Philippe Huneman, 19-41. Dordrecht: Springer Netherlands. https://doi. org/10.1007/978-94-007-5304-4_2.

. 2015. "Entrenchment as a Theoretical Tool in Evolutionary Developmental Biology." In Conceptual Change in Biology: Scientific and Philosophical Perspectives on Evolution and Development, edited by Alan C. Love, 365-97. Springer. https://doi.org/10.1007/978-94-017-9412-1.

Winsor, Mary P. 2006. "The Creation of the Essentialism Story: An Exercise in Metahistory.” History and Philosophy of the Life Sciences 28 (2): 149-74.

Witteveen, Joeri. 2015. “'A Temporary Oversimplification’: Mayr, Simpson, Dobzhansky, and the Origins of the Typology/Population Dichotomy (Part 1 of 2).” Studies in History and Philosophy of Science Part C: Studies in History and Philosophy of Biological and Biomedical Sciences 54: 20-33. https://doi.org/10.1016/j.shpsc.2015.09.007.

—. 2016. “'A Temporary Oversimplification': Mayr, Simpson, Dobzhansky, and the Origins of the Typology/Population Dichotomy (Part 2 of 2).” Studies in History and Philosophy of Science Part C: Studies in History and Philosophy of Biological and Biomedical Sciences 57: 96-105. https: T/doi.org/10.1016/j.shpsc.2015.09.006.

Wouters, Arno G. 2005. “The Function Debate in Philosophy.” Acta Biotheoretica 53 (2): 123-51. https://doi.org/10.1007/s10441-005-5353-6.

- 2007. "Design Explanation: Determining the Constraints on What Can Be Alive." Erkenntnis 67 (1): 65-80. https://doi.org/10.1007/s10670-007-9045-2.

Wright, Sewell. 1963. "Adaptation and Selection." In Genetics, Paleontology and Evolution, edited by Glenn L. Jepsen, George Gaylord Simpson, and Ernst Mayr, 365-89. New York: Atheneum.

(C) 2019 Author(s)

This is an open-access article distributed under the terms of the Creative Commons Attribution 4.0 International license, which permits anyone to download, copy, distribute, display, or adapt the text without asking for permission, provided that the creator(s) are given full credit.

ISSN 2475-3025 